\title{
Discovery of an unusually compact lensed Lyman Break Galaxy from Hyper Suprime-Cam Survey
}

\author{
Anton T. Jaelani, 1,2 Anupreeta More, ${ }^{3,4}$ Alessandro Sonnenfeld, ${ }^{3,5}$ Masamune Oguri, ${ }^{3,6,7}$ \\ Cristian E. Rusu, ${ }^{8}$ Kenneth C. Wong, ${ }^{3}$ James H. H. Chan, ${ }^{9}$ Sherry H. Suyu, ${ }^{10,11,12}$ \\ Issha Kayo, ${ }^{13}$ Chien-Hsiu Lee, ${ }^{14}$ Kaiki T. Inoue ${ }^{1}$ \\ ${ }^{1}$ Department of Physics, Kindai University, 3-4-1 Kowakae, Higashi-Osaka, Osaka 577-8502, Japan \\ ${ }^{2}$ Astronomical Institute, Tohoku University, 6-3 Aramaki, Aoba-ku, Sendai 980-8578, Japan \\ ${ }^{3}$ Kavli Institute for the Physics and Mathematics of the Universe (IPMU), 5-1-5 Kashiwanoha, Kashiwa-shi, Chiba 277-8583, Japan \\ ${ }^{4}$ The Inter-University Centre for Astronomy and Astrophysics, Post Bag 4, Ganeshkhind, Pune, 411007, India \\ ${ }^{5}$ Leiden Observatory, Leiden University, Niels Bohrweg 2, 2333 CA Leiden, Netherlands \\ ${ }^{6}$ Department of Physics, The University of Tokyo, 7-3-1 Hongo, Bunkyo-ku, Tokyo 113-0033, Japan \\ ${ }^{7}$ Research Center for the Early Universe, The University of Tokyo, 7-3-1 Hongo, Bunkyo-ku, Tokyo 113-0033, Japan \\ ${ }^{8}$ Subaru Telescope, National Astronomical Observatory of Japan, 2-21-1 Osawa, Mitaka, Tokyo 181-0015, Japan \\ ${ }^{9}$ Laboratoire d'Astrophysique, Ecole Polytechnique Fédérale de Lausanne (EPFL), Observatoire de Sauverny, CH-1290 Versoix, Switzerland \\ ${ }^{10}$ Max-Planck-Institut für Astrophysik, Karl-Schwarzschild-Straße 1, 85748 Garching, Germany \\ ${ }^{11}$ Institute of Astronomy and Astrophysics, Academia Sinica (ASIAA), 11F of ASMAB, No. 1, Section 4, Roosevelt Road, Taipei 10617, Taiwan \\ ${ }^{12}$ Physik-Department, Technische Universität München, James-Franck-Straße 1, 85748 Garching, Germany \\ ${ }^{13}$ Department of Liberal Arts, Tokyo University of Technology, Ota-ku, Tokyo 144-8650, Japan \\ ${ }^{14}$ National Optical Astronomy Observatory (NOAO) 950 N Cherry Avenue, Tucson, AZ 85719, USA
}

Accepted XXX. Received YYY; in original form ZZZ

\begin{abstract}
We report a serendipitous discovery of HSC J0904-0102, a quadruply lensed Lyman Break Galaxy (LBG) in the Survey of Gravitationally Lensed Objects in Hyper Suprime-Cam Imaging (SuGOHI). Owing to its point-like appearance, the source was thought to be a lensed active galactic nucleus. As a result, we obtained followup spectroscopic data with the Gemini Multi-Object Spectrographs on Gemini South Telescope which confirmed this to be a lens system. The deflecting foreground galaxy is a typical early-type galaxy but at a high redshift of $z_{\ell}=0.957$ with stellar velocity dispersion $\sigma_{v}=259 \pm 56 \mathrm{~km} \mathrm{~s}^{-1}$ and the lensed source turned out to be an LBG at $z_{\mathrm{s}}=3.403$, based on the sharp drop bluewards of Ly $\alpha$ and other absorption features. A simple lens mass model for the system assuming a Singular Isothermal Ellipsoid yields an Einstein radius of $\theta_{\text {Ein }}=1.23^{\prime \prime}$, a total mass within the Einstein radius for the deflector of $M_{\text {Ein }}=(5.55 \pm 0.31) \times 10^{11} M_{\odot}$ corresponding to the velocity dispersion of the deflector of $\sigma_{\text {SIE }}=283 \pm 4 \mathrm{~km} \mathrm{~s}^{-1}$, which is in good agreement with the value derived spectroscopically, and a magnification factor for more isolated lensed LBG image of $\sim$ 6.5. After comparing with other lensed LBGs and typical $z \sim 4$ LBG populations, our LBG is unusually compact, an outlier of over $2 \sigma$. Together with a previously discovered SuGOHI lens (More et al. 2017) that had similarly highly compact nature, we believe that the HSC Survey is extending LBG studies down to smaller sizes.
\end{abstract}

Key words: galaxies: high-redshift - gravitational lensing: strong.

\section{INTRODUCTION}

Strong gravitational lensing has proven to be a powerful tool for constraining mass distributions of galaxies and clusters, cosmology and in-depth study of high redshift sources such

^ Contact e-mail: anton@phys.kindai.ac.jp as galaxies or quasars. Strongly lensed sources allow detailed follow-up studies at a fraction of the telescope time that would be necessary for unlensed sources (e.g., Pettini et al. 2002; Stark et al. 2007; Quider et al. 2009, 2010; Richard et al. 2008, 2011).

The observed properties of Lyman Break Galaxies (LBGs) provide important constraints in our understand- 

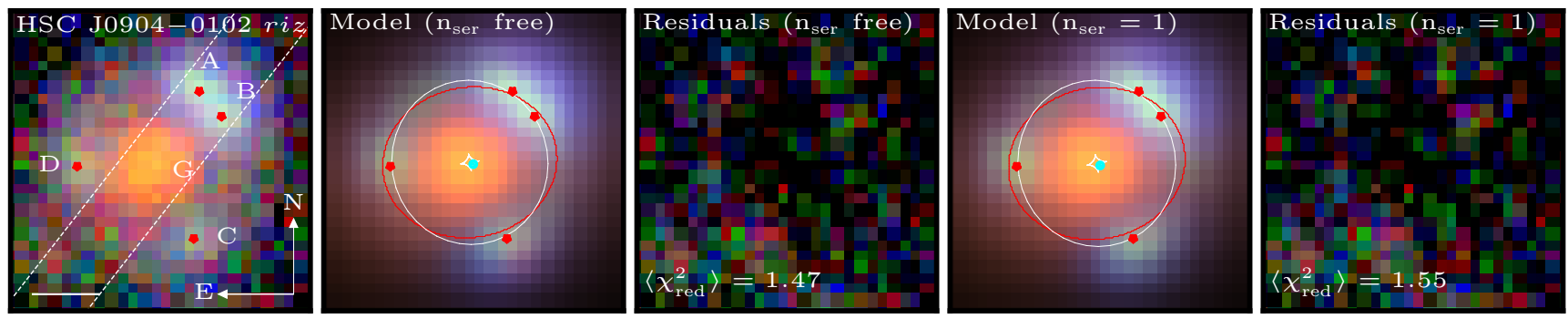

Figure 1. First (left-most): HSC riz composite image shows the location of quadruply lensed images (A, B, C, D) and the position of the early-type galaxy (G). Surface brigthness peaks of the lensed galaxy are indicated by red dots. White dashed lines indicate slit position used in the spectroscopic observations. North is up and East is left. The white solid bar shows a scale of $1^{\prime \prime}$. Second : Model riz composite image generated from lens mass modelling of the lens system, where the source is assumed to be an extended galaxy following a Sérsic profile (with free Sérsic index). The best fit index comes out to be $n_{\text {ser }} \sim 3$. This panel also shows the unlensed source position (cyan dot). The red contours show the caustics in the source plane and white contours mark the corresponding critical curves in the image plane indicating regions of extremely high magnification. Third : the residual composite image by subtracting the model from the data. Fourth : Same as the second panel, but for fixed Sérsic index, $n_{\text {ser }}=1$. Fifth (right-most) : Same as the third panel, but for fixed Sérsic index, $n_{\text {ser }}=1$.

ing of galaxy formation scenarios. In star forming galaxies at $z \sim 3$, LBGs spectra will show a "break" at around the Lyman limit (912 $\AA$ ) in the spectral energy distribution (SED). However, LBGs are generally faint, which makes it difficult to obtain the spectral properties of individual LBGs, so many hundreds are needed to examine the general properties e.g. the redshift distribution (Adelberger et al. 1998, 2003; Steidel et al. 1998), the rest-frame UV spectroscopic properties (Shapley et al. 2003), slope of the UV luminosity function (Reddy \& Steidel 2009), and morphology and size evolution (Shibuya et al. 2015, henceforth, S15).

The additional magnification provided by strong gravitational lensing enables more detailed investigations of properties of LBGs. Several lensed LBGs have now been found and studied, e.g. the interstellar medium of the first lensed LBG MS 1512-cB58 (cB58, Yee et al. 1996; Pettini et al. $2000,2002)$ which gives a hint about when most of the metal enrichment occurred. Further studies of cB58 with Spitzer by Siana et al. (2009) have reported that the UV-inferred star formation rate is lower than measured in the IR by a factor of 3-5. Baker et al. (2004) found the first direct evidence of the existence of a sizeable cold gas reservoir in an LBG by using observed CO emission of cB58. Similar studies of "Cosmic Eye" were carried out (Smail et al. 2007; Coppin et al. 2007). Arc-like images of LBGs, lensed by massive galaxy clusters, have been discovered e.g. A2218-384 (Ebbels et al. 1996), 1E0657-56 (Mehlert et al. 2001), the Sextet Arcs (Frye et al. 2007), SGAS J122651.3+215220 and SGAS J152745.1+065219 (Koester et al. 2010). However, only few additional strongly lensed LBGs have been lensed by a single massive galaxy, e.g. the Einstein Ring (Cabanac et al. 2005), the Einstein cross (Bolton et al. 2006), the Cosmic Horseshoe (Belokurov et al. 2007), and the 8 o'clock arc (Allam et al. 2007). Studies of the current and future lensed LBGs samples will give us a better insight when drawing general conclusions for certain properties of a whole LBG population. So, adding even one lensed LBG would be of tremendous value.

In this paper, we report the discovery and spectroscopic confirmation of a quadruply lensed LBG from the Hyper Suprime-Cam Subaru (HSC) Survey (Aihara et al. 2018a).
Our paper is organized as follows. In Section 2, we describe the discovery of HSC J0904-0102. We present the spectroscopic follow-up observation on this system, and reduction procedure in Section 3. In Section 4, we describe the HSC imaging data analysis and the redshift determinations for the lens and the source. In Section 5, we describe the lens mass modelling for the system. We calculate and discuss the properties of the delensed source in Section 6. We present our conclusions in Section 7. Throughout the paper, we use $\Omega_{\mathrm{m}}=0.27, \Omega_{\Lambda}=0.73$ and $H_{0}=73 \mathrm{~km} \mathrm{~s}^{-1} \mathrm{Mpc}^{-1}$. All quoted magnitudes are in the $\mathrm{AB}$ system, all position angles are measured East of North and all uncertainties are $1 \sigma$ and are to be Gaussian.

\section{DISCOVERY OF HSC J0904-0102}

The HSC Survey is an ongoing imaging survey, expected to cover about $1400 \mathrm{deg}^{2}$ area in five bands $(g, r, i, z$ and $y)$ down to $r \sim 26$ with Hyper Suprime-Cam (Miyazaki et al. 2018; Komiyama et al. 2018; Kawanomoto et al. 2018; Furusawa et al. 2018), a wide-field (1.7 degree diameter) optical camera installed on the $8.2 \mathrm{~m}$ Subaru Telescope. The data are processed with hscPipe, which is derived from the Large Synoptic Survey Telescope pipeline (Axelrod et al. 2010; Jurić et al. 2017; Ivezić et al. 2008, 2019). We used photometric data from the HSC Wide S17A internal data release of the HSC survey for our analysis. The seeing in the HSC- $g, r, i, z$ and $y$ images is found to be $0.62^{\prime \prime}, 0.76^{\prime \prime}, 0.49^{\prime \prime}, 0.57^{\prime \prime}$ and $0.59^{\prime \prime}$, respectively.

HSC J0904-0102 was discovered serendipitously during the visual inspection of galaxy clusters selected from the HSC Survey (CAMIRA clusters, for details see Oguri et al. 2018, $232 \mathrm{deg}^{2}$ of the HSC Wide S16A internal data release). This lens system comprises of a lens galaxy $(\mathrm{G})$ and quadruply lensed images (A, B, C, D) of the LBG (see left panel of Figure 1). HSC J0904-0102 is thus part of lens samples discovered from HSC, namely, the Survey of Gravitationally lensed Objects in HSC Imaging (SuGOHI, Sonnenfeld et al. 2018; Wong et al. 2018; Sonnenfeld et al. 2019, Jaelani et al. in prep., Chan et al. in prep.). 

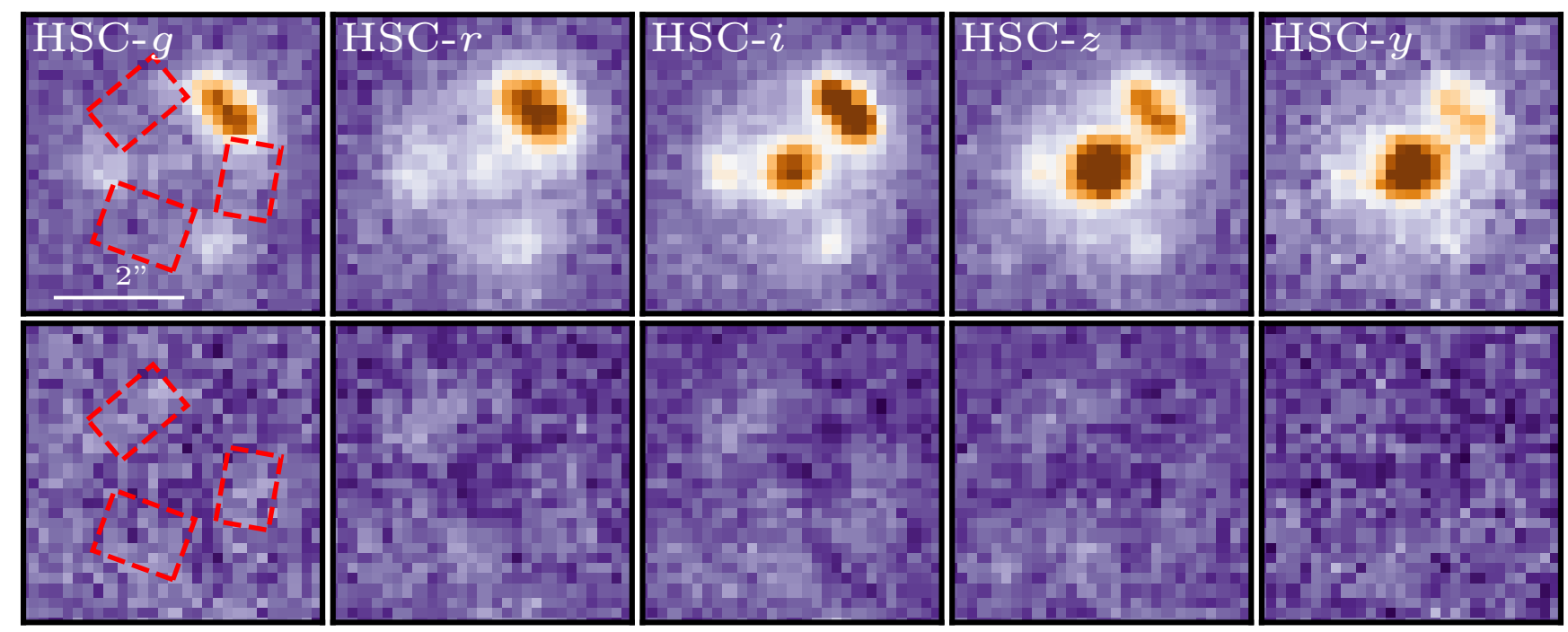

Figure 2. GALfiT modelling results in HSC grizy images for Sérsic source model. Top panels show the HSC data, whereas the bottom panels show GALFIT model-subtracted residual images in the respective bands. Images are $\sim 5^{\prime \prime}$ on the side. The bar shows a scale of $2^{\prime \prime}$. Red boxes show the location of very faint extended bluish emission most probably arising from the lensed source consistent with the bluish color of the source.

Table 1. Coordinates, photometric redshifts (uncertainties), magnitudes (uncertainties) in the HSC grizy data from fitting Sérsic profile using Galfit.

\begin{tabular}{ccccccccc}
\hline $\begin{array}{c}\text { RA } \\
(\mathrm{J} 2000.0)\end{array}$ & $\begin{array}{c}\text { Decl. } \\
\text { (J2000.0) }\end{array}$ & Name & $\begin{array}{c}z_{\text {phot }} \\
\text { err }\end{array}$ & $\begin{array}{c}g \\
\text { err }\end{array}$ & $\begin{array}{c}r \\
\text { err }\end{array}$ & $\begin{array}{c}i \\
\text { err }\end{array}$ & $\begin{array}{c}z \\
\text { err }\end{array}$ & $\begin{array}{c}y \\
\text { err }\end{array}$ \\
\hline 09:04:29.75 & $-01: 02: 28.26$ & G & $1.01(0.05)$ & $25.13(0.11)$ & $23.12(0.12)$ & $21.91(0.11)$ & $20.88(0.06)$ & $20.59(0.04)$ \\
09:04:29.71 & $-01: 02: 27.14$ & A & $3.26(0.09)$ & $24.13(0.04)$ & $23.19(0.03)$ & $23.01(0.03)$ & $22.94(0.05)$ & $22.81(0.09)$ \\
09:04:29.68 & $-01: 02: 27.53$ & B & $3.44(0.05)$ & $23.91(0.04)$ & $23.02(0.03)$ & $22.92(0.04)$ & $22.87(0.05)$ & $22.79(0.09)$ \\
09:04:29.71 & $-01: 02: 29.47$ & C & $3.50(0.04)$ & $24.88(0.09)$ & $24.03(0.05)$ & $23.95(0.07)$ & $23.84(0.11)$ & $23.61(0.13)$ \\
09:04:29.82 & $-01: 02: 28.34$ & D & $3.49(0.07)$ & $25.12(0.10)$ & $24.28(0.06)$ & $24.08(0.07)$ & $24.02(0.13)$ & $23.97(0.13)$ \\
\hline Total LBG & & & & $22.89(0.03)$ & $22.00(0.02)$ & $21.86(0.02)$ & $21.79(0.04)$ & $21.68(0.05)$ \\
\hline
\end{tabular}

\section{SPECTROSCOPIC FOLLOW-UP OBSERVATIONS}

In order to confirm the lensing effect and study the nature of the source galaxy, we performed follow-up spectroscopic observation of HSC J0904-0102 on 2017 November 18 (GS2017B-FT-5, PI: A. T. Jaelani) with Gemini Multi-Object Spectrographs (GMOS) on Gemini South Telescope via the Fast Turnaround program (Mason et al. 2014). The seeing was around $1.0^{\prime \prime}-1.4^{\prime \prime}$. GMOS has a wavelength coverage from $4000 \AA$ to $8000 \AA$ at a spectral resolution of $R=1918$ in the long-slit mode with R400-G5305 grating and GG455 blocking filter. A slit of $1^{\prime \prime}$ was placed along the merging pair of images and lens galaxy with position angle P.A. $=-29^{\circ}$ (see dashed lines in Figure 1). The total exposure time was 40 minutes. Based on our photometric redshift estimates (see details in Section 4), we set spatial and spectral dithering with two different central wavelengths (7000 $\AA$ and $7100 \AA$ ) to avoid any important lines from falling on the gaps between the detectors. The data were binned $2 \times 2$ giving spectral dispersion $1.48 \AA$ pixel ${ }^{-1}$.

We used the Image Reduction and Analysis Facility $(\text { IRAF })^{1}$ v2.16 (Tody 1986, 1993) with the GMos package v1.13 (Gemini Observatory \& AURA 2016) and PyTHON tasks $\left(\mathrm{PYRAF}^{2}\right)$ to reduce the spectra. In general, we followed standard procedure in which the spectra were bias subtracted, flat fielded and sky subtracted using the tasks gsflat and gsreduce. We used CuAr lamp and spectroscopic standard star to calibrate the wavelength and flux, respectively (using the tasks gswavelength and gstransform for transformation), then coadded the dithered frames. By using gsextract in an iterative mode, we selected optimal size and position to extract the lens galaxy and background source image spectra. The one-dimensional (1D) spectra of the lens galaxy and lensed images were smoothed with a box 1D convolution kernel of $3.7 \AA$.

1 IRAF is distributed by the National Optical Astronomy Observatory, which is operated by the Association of Universities for Research in Astronomy (AURA) under a cooperative agreement with the National Science Foundation.

2 PyRAF is a product of the Space Telescope Science Institute, which is operated by AURA for NASA 


\section{DATA ANALYSIS}

\subsection{Imaging}

We modelled and analysed the multi-band HSC imaging data for HSC J0904-0102 using GALFIT (Peng et al. 2002) to measure the positions and magnitudes of the lens galaxy and the lensed images. We used the PSF PICKER tool to generate PSF models in all of the bands $(g, r, i, z$ and $y)$ of the HSC survey for the Wide Field Survey described in Aihara et al. (2018b, 2019). We find that the lens galaxy in $i$-band is well fit by a model with fixed Sérsic index $n_{\text {ser }}=4$, ellipticity $e=0.39$, position angle P.A. $=-78^{\circ}$ and effective radius (half-light radius measured along the major axis), $r_{e}=0.71^{\prime \prime}$ corresponding to $R_{e} \sim 5.49 \mathrm{kpc}$ in the physical scale. While the lens galaxy was modelled with a single Sérsic profile, we tested different models for the lensed images - a PSF model, assuming that the lensed source is point-like, and a Sérsic profile, assuming it is extended.

We first modelled the $i$-band data and used this best-fit model as an initial model when fitting the profile for the lens galaxy and the lensed source in the rest of the bands. In Figure 2, we show all of the multi-band data from HSC and the model-subtracted residual images for the respective bands for Sérsic source model. The extended ring-like emission of the underlying host galaxy is revealed in some of the residuals. In general, we found that both choices of source models fit the data equally well, suggesting that the source is highly compact. Measured magnitudes for single Sérsic model of the lens galaxy and four separate Sérsic model of the lensed images are given in Table 1.

Prior to obtaining spectroscopic follow-up data, we also estimated photometric redshifts for the lens galaxy and the lensed images using the publicly available spectral energy distribution (SED) fitting code LEPhARE (Arnouts et al. 1999; Ilbert et al. 2006). We used galaxy templates from Bruzual \& Charlot (2003), subsequently modified by LEPHARE with variable extinction laws (Calzetti et al. 1994) and addition of emission lines following classical recipes that relate galaxy star formation rates and luminosities in the UV continuum, recombination lines and forbidden lines (Kennicutt 1998). The source redshift seems to be broadly consistent with the expected redshift $z \sim 3$, from the $g-r$ vs. $r-i$ colors (e.g. Figure 4 of Ono et al. 2018). The redshifts thus estimated are given in Table 1.

\subsection{Spectroscopy}

We show the reduced spectra of the lens and the source galaxy in Figure 3. For comparison, we also show composite spectra for early-type galaxy and LBGs from Dobos et al. (2012) and Shapley et al. (2003), respectively.

We assigned the redshifts of the lens and the source galaxy by identifying a set of lines at a common redshift, by fitting a Gaussian profile to each line in order to determine their central wavelength and taking the mean redshift of the entire set of lines. We measured the redshift of the lens galaxy to be $z_{\ell}=0.957 \pm 0.003$ based on a strong continuum break around $7800 \AA$ and characteristic $\mathrm{K}$ and $\mathrm{H}, \mathrm{H} \delta$ and $\mathrm{G}$ band present in early-type galaxies. From these lines, we measured a central velocity dispersion $\sigma_{v}=259 \pm 56 \mathrm{~km} \mathrm{~s}^{-1}$ using the Penalized Pixel Fitting (PPXF) code of Cappellari (2017), with stellar templates for the fit, we had 100 selected stars (F, G, K, M) from the MILES stellar template library of Sánchez-Blázquez et al. (2006). The mass of the lens galaxy estimated from the measured velocity dispersion $\sigma_{v}$ and the effective radius $r_{e}$ using Equation 2 from Wolf et al. $(2010)$ is $M_{\text {gal }}=(3.4 \pm 1.5) \times 10^{11} M_{\odot}$.

Finally, the source galaxy is confirmed at $z_{\mathrm{s}}=$ $3.403 \pm 0.001$ from absorption lines identification of SiII $\lambda \lambda \lambda(1260.4,1304.4,1526.7) \AA$, OI $\lambda 1302.2 \AA$, Cіл $\lambda 1334.5$ $\AA$, Sirv $\lambda \lambda(1393.8,1402.8) \AA$, CIv $\lambda \lambda(1538.2,1553.8) \AA$, FeII $\lambda 1608.5 \AA$, Aliı $\lambda 1670.8 \AA$, Aliı $\lambda \lambda(1854.7,1862.8) \AA$ and Cir $\lambda 1908.7 \AA$. The absorption features and a sharp drop in the flux of the $\operatorname{Ly} \alpha$ in the source galaxy spectrum are strong evidences of an LBG galaxy (Steidel et al. 2004).

\section{LENS MODELLING}

We carried out lens mass modelling using the publicly available lens modelling software GLAFIC (Oguri 2010) to infer the properties of the lens galaxy and the source and to see if there is any improvement to the fits with GALFIT. We used the information from each pixel of the multi-band HSC images within a $5^{\prime \prime}$ box centered on the lens galaxy as our input data constraints for the mass model.

Light from the lens and source galaxy are each modelled with a seven-parameter elliptical Sérsic profile. In order to subtract the lens galaxy G, we used the best-fit of GALFIT as initial parameters in the GLAFIC input. For the source galaxy, we let every parameter of the Sérsic profile vary: magnitude, position $\left(x_{\mathrm{s}}, y_{\mathrm{s}}\right)$, position angle, ellipticity, effective radius and index.

The mass distribution of the lens galaxy is modelled with a Singular Isothermal Ellipsoid (SIE) profile which has five parameters, namely, centroid ( $x$ and $y$ ), mass, ellipticity and position angle. We fixed the center of mass to that of the center of the light profile of the lens galaxy and vary the velocity dispersion $\sigma_{\mathrm{SIE}}$ (proxy for mass), ellipticity (e) and position angle. HSC J0904-0102 system is located $\sim 195^{\prime \prime}$ (corresponding to $\sim 1500 \mathrm{kpc}$ in the physical scale) away from a cluster of galaxies at $z=0.864$ with a richness of $\sim 10$. Therefore, we believe that the external convergence and shear arising due to the cluster is negligible. We run Monte Carlo Markov Chain (MCMC) using EMCEE (Foreman-Mackey et al. 2013) to sample the posterior distribution of the parameters. The best model is used to predict $\theta_{\text {SIE }}$, the Einstein radius, defined as

$\theta_{\mathrm{SIE}}=4 \pi\left(\frac{\sigma_{\mathrm{SIE}}}{c}\right)^{2} \frac{D_{\mathrm{ls}}}{D_{\mathrm{S}}}$

where $D_{\mathrm{s}}$ is the angular diameter distance of the source from the observer, $D_{\mathrm{ls}}$ is the angular diameter distance between the lens and the source and $c$ is the speed of light.

The best-fit lens model is shown in the second panel of Figure 1 (with reduced $\chi^{2}=1.25,1.67,1.08,1.66,1.72$ for $g, r, i, z, y$-bands, respectively) and the inferred best-fit parameters for the lens and the source are reported in Table 2. The number of degrees of freedom (DOF) is the number of pixels minus the number of the lens and the source parameters used in fitting thus $N_{\mathrm{DOF}}=444$. We found an effective radius of the source $r_{e}=0.022^{\prime \prime}$ corresponding to $R_{e} \sim 0.16$ kpc with Sérsic index $n_{\text {ser }} \sim 3$. In order to check the robustness of the source size, we also re-ran MCMC of the lens 


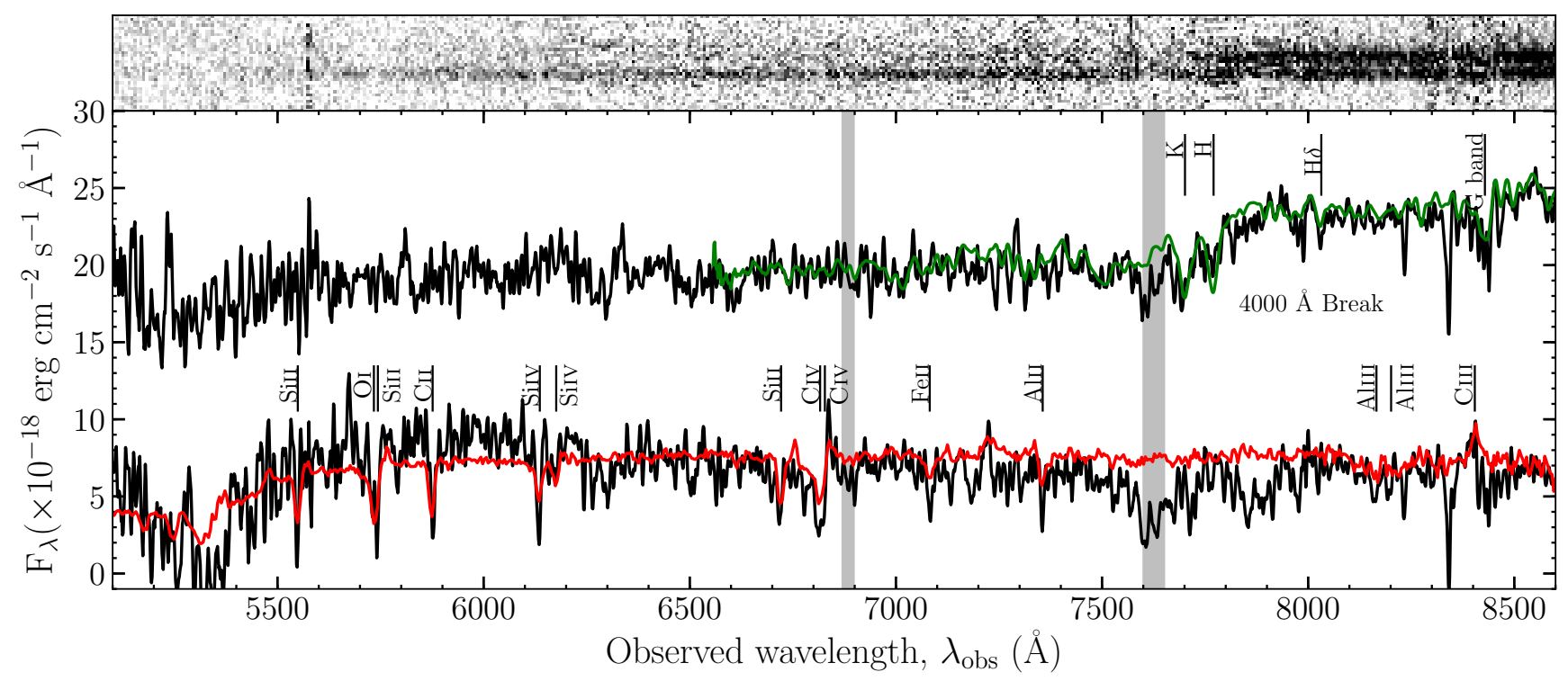

Figure 3. Top panel: 2D spectrum of lens (upper trace) and lensed galaxy (lower trace) from Gemini GMOS spectroscopic follow-up observation. Bottom panel: 1D spectrum of the lens (upper curve) and lensed galaxy (lower curve) superposed with composite early-type galaxy spectrum (Dobos et al. 2012) in green and composite LBG spectrum (Shapley et al. 2003) in red for comparison, respectively, shifted by the measured redshifts. Absorption lines are labelled according to the redshifts of the lens galaxy, $z \ell=0.957$ and lensed galaxy, $z_{\mathrm{s}}=3.403$.

Table 2. Top: Best-fit model parameters in $i$-band with $1 \sigma$ errors from MCMC. Bottom: Source centroid is relative to the lens centroid. Lens and source quantities.

\begin{tabular}{lc}
\hline \hline Lens Parameter & Best-fit \\
\hline Velocity dispersion, $\sigma_{\text {SIE }}\left(\mathrm{km} \mathrm{s}^{-1}\right)$ & $283 \pm 4$ \\
Ellipticity, $e$ & $0.15 \pm 0.01$ \\
Position Angle (degree) & $-80 \pm 2$ \\
\hline Source Parameter & \\
\hline Magnitude, $i$ & $25.98 \pm 0.09$ \\
Ellipticity, $e$ & $0.55 \pm 0.04$ \\
Position Angle (degree) & $-35 \pm 3$ \\
Effective radius, $r_{\mathrm{e}}(\operatorname{arcsec})$ & $0.022 \pm 0.007$ \\
Sérsic index, $n_{\text {ser }}$ & $2.98 \pm 0.25$ \\
\hline \hline Quantities & Values \\
\hline$\Delta \mathrm{x}$ (arcsec) & $0.04 \pm 0.01$ \\
$\Delta \mathrm{y}(\operatorname{arcsec})$ & $-0.03 \pm 0.01$ \\
Spectroscopic lens redshift, $z_{\ell}$ & $0.957 \pm 0.003$ \\
Spectroscopic source redshift, $z_{\mathrm{s}}$ & $3.403 \pm 0.001$ \\
Einstein Radius, $\theta_{\text {Ein }}(\operatorname{arcsec})$ & $1.23^{\prime \prime} \pm 0.04^{\prime \prime}$ \\
Mass within $\theta_{\text {Ein }}, M_{\text {Ein }}\left(\boldsymbol{M}_{\odot}\right)$ & $5.55 \pm 0.31 \times 10^{11}$ \\
\hline
\end{tabular}

model assuming fixed Sérsic index $n_{\text {ser }}=1$ and found similar results with free Sérsic index. We also found that the source magnitude, $i=25.98 \pm 0.12$, for $n_{\mathrm{ser}}=1$ was consistent within $1 \sigma$ magnitude for free Sérsic index.

\section{DISCUSSION}

In this section, we study and explore the reasons that might have contributed to the detection of a system like HSC
J0904-0102 including any biases from strong lensing and the robustness of the inferred compactness, by testing for systematic errors introduced due to lens modelling.

\subsection{Intrinsic source properties}

Having determined the lensing magnification and effective radius of the $\mathrm{LBG}$, we can estimate its intrinsic luminosity and size. We calculate the absolute UV (at rest-frame 1700 $\AA$ ) magnitude from the apparent magnitude of the source and redshift $z_{s}$ using

$M_{\mathrm{UV}}=m+2.5 \log \left(1+z_{s}\right)-5 \log \left(\frac{D_{L}\left(z_{s}\right)}{10 \mathrm{pc}}\right)+\left(m_{\mathrm{UV}}-m\right)$

where $D_{L}$ is the luminosity distance in the unit parsec, $m$ is the apparent magnitude and $\left(m_{\mathrm{UV}}-m\right)$ is the $k$-correction term between the magnitude at rest-frame UV and the observed magnitude that we use. In order to correct for reddening by dust extinction, we estimated the $k$-correction term by assuming the intrinsic UV continuum slope from Figure 3 in Sawicki \& Thompson (2006). Using the HSC Wide S17A $i$-band magnitude of the quadruple images, we determine a rest-frame $1700 \AA$ absolute magnitude $M_{1700}=-23.86 \pm 0.02$ corresponding to luminosity $L_{1700} \sim 1.53 \times 10^{30} \mathrm{ergs} \mathrm{s}^{-1} \mathrm{~Hz}^{-1}$ which translates into a star formation rate of $\sim 210 M_{\odot} \mathrm{yr}^{-1}$, by adopting a Salpeter IMF with an upper mass cutoff 100 $M_{\odot}$ (Kennicutt 1998), and without any correction for reddening or magnification.

Correcting for the estimated magnification and UV reddening, we determine absolute UV magnitude using two different $k$-correction: the 100 and $10 \mathrm{Myr}$ old continuously star-forming template with a dust attenuation of $E(B-V)=0.2$ and 0.0 , respectively. For $k$-correction with $[100 \mathrm{Myr}, \mathrm{E}(\mathrm{B}-\mathrm{V})=0.2]$, we determine $M_{1700}=-19.73 \pm 0.09$ 


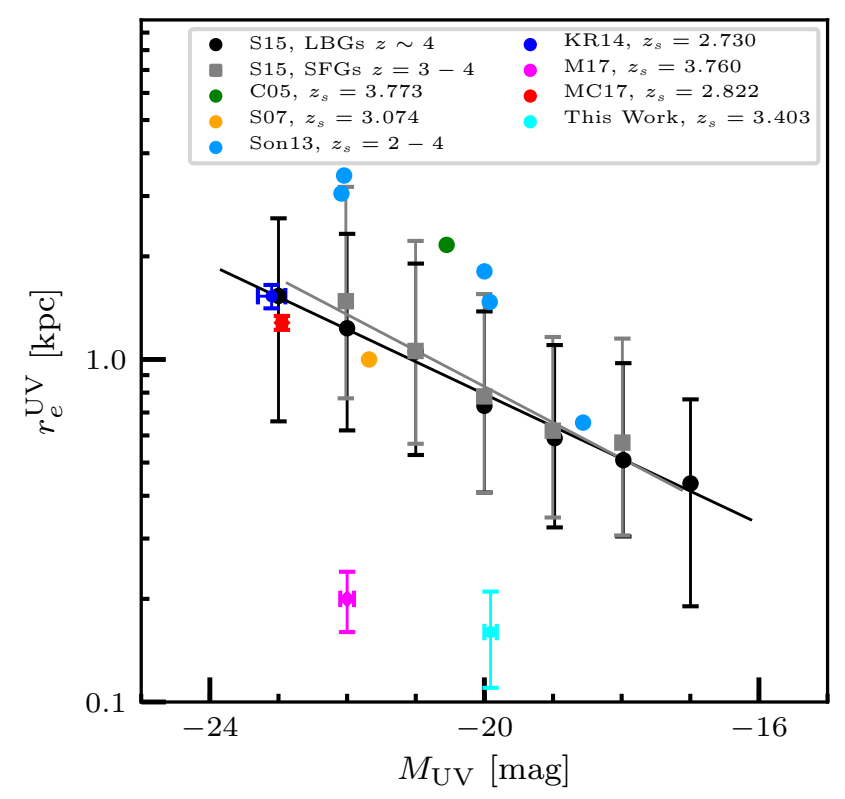

Figure 4. Effective radius and absolute UV magnitude. HSC J0904-0102 is shown along with another lensed LBG or LAE systems at redshift $z \sim 3$ from Cabanac et al. (2005, C05), Smail et al. (2007, S07), Sonnenfeld et al. (2013a,b, Son13), KostrzewaRutkowska et al. (2014, KR14), More et al. (2017, M17), and Marques-Chaves et al. (2017, MC17). For comparison, we show the size-luminosity relation of LBGs (black line) and star forming galaxy (SFGs, grey line) from S15. The line denotes their best-fit for the $r_{e}-M_{\mathrm{UV}}$ relation and their data points with error bars indicate the median $r_{e}$ and the 16 th and 84 th percentiles for their sample.

corresponding to $L_{1700} \sim 0.34 \times 10^{29} \mathrm{ergs} \mathrm{s}^{-1} \mathrm{~Hz}^{-1}$ and $M_{1700}=-19.91 \pm 0.09$ corresponding to $L_{1700} \sim 0.40 \times 10^{29}$ $\operatorname{ergs~s}^{-1} \mathrm{~Hz}^{-1}$ for $k$-correction with [10 Myr, $\left.\mathrm{E}(\mathrm{BV})=0.0\right]$. We derive an intrinsic star formation rate of $\sim 5 M_{\odot} \mathrm{yr}^{-1}$. HSC J0904-0102 is intrinsically less luminous relative to typical luminosity of LBG, $L_{\mathrm{UV}}=1.06 \times 10^{29} \mathrm{ergs} \mathrm{s}^{-1} \mathrm{~Hz}^{-1}$ which correspond to $m_{\mathrm{UV}}(\simeq 1500-1700 \AA)=24.61$ (Reddy \& Steidel 2009).

Following the same approach as More et al. (2017), we estimate the true source magnitude and size in a manner complementary to the method described in Section 5. Using the magnification factor of image $\mathrm{C}$, which is more isolated and brighter, from the lens model, $\mu_{\mathrm{C}} \sim 6.5$, we obtain a delensed source magnitude of $i=25.98$. This result was consistent within $1 \sigma$ magnitude for the best-fitting source magnitude thus obtained $i=25.98 \pm 0.09$. The true source size is estimated to be $r_{e, t}=\sqrt{r_{e}^{2} \text {, Galfit } / \mu_{\mathrm{C}}}=0.02^{\prime \prime} \pm 0.01^{\prime \prime}$ where $r_{e}$, Galfit $=0.05^{\prime \prime} \pm 0.02^{\prime \prime}$, corresponds to $R_{e, t} \sim 0.14 \pm 0.06 \mathrm{kpc}$ in physical units.

Our estimated size from both methods GLAFIC and GALFIT suggests a size much smaller than the typical LBGs at $z \sim 4$ and star-forming galaxies (SFGs) at $z \sim 3-4$ (see Figure 4 and S15). The SFGs and LBGs in S15 are identified from deep HST surveys (e.g. HUDF, CANDELS and HFF ${ }^{3}$ )

3 Hubble Ultra Deep Field, Cosmic Assembly Near-infrared Deep Extragalactic Legacy Survey, Hubble Frontier Field and are apparently faint sources (optical/UV mag $~ 23-28$ for redshifts spanning $z \sim 0-10$ ). Even though detected with HST resolution, these sources are bigger than our lensed LBG. On the other hand, the combined imaging area investigated in S15 is much smaller (a few sq. deg.) compared to HSC Survey (hundreds of sq. deg. in this work). Thus, it is possible that the work of S15 is missing highly compact LBGs, such as our source, if they come from a rarer population.

In Figure 4, we show another quad, lens HSC J1152+0047 (More et al. 2017, henceforth, referred to as J1152), also discovered serendipitously from HSC. The J1152 quad also happens to be unusually compact compared to the rest of the LBGs or Lyman Alpha Emitters (LAEs) at $z \sim 4$. Additionally, we compare with other lensed LBGs from the literature, lying at $z \sim 2-4$, for which we could derive the sizes and magnitudes. Their sizes and absolute magnitudes are consistent with the typical LBG population of S15. A few others that could not be added here (due to missing sizes or absolute magnitudes) for a quantitative comparison, showed fairly extended arc-like emission suggesting that the intrinsic sources are unlikely to be highly compact. Overall, we did not find any lensed LBGs in the literature as compact as our source and J1152.

Similar to J1152, our lens system is also located in the footprint of other surveys such as the KiDS (Kuijken et al. 2019), PanSTARRS (Chambers et al. 2016), and SDSS (DR15 Aguado et al. 2019) but is barely detectable or identifiable as a lens owing to the shallower depth of those surveys coupled with the faintness of the lens system (see panels on the left in Figure 5). We then show how the appearance of lensed images would change in HSC imaging if the source were to be a typical galaxy following the size-magnitude relation of S15. First, we fix the source magnitude to the best-fit model and estimate the source size (following S15). This makes the source bigger (top-right panels) and hence, harder to detect in HSC imaging owing to decreased surface brightness. Next, we fix the source size to determine its magnitude (following S15). This makes the source fainter (bottom-right panels) and yet again, impossible to detect in HSC imaging. For reference, we show the best-fit source model (middle-right panels) in Figure 5.

Since Sérsic index can affect size and magnitude parameters, we also tested models by varying the Sérsic index parameter $\left(n_{\mathrm{ser}}=1\right.$ or free). We find that the data is not able to constrain the Sérsic index accurately but the inferred source size is measured more robustly. Finally, we note that differential magnification due to lensing may create a bias in favor of the detection of more compact sources than their bigger counterparts (Hezaveh et al. 2012; Oldham et al. 2017). While this may be important, we believe that lensing bias may not be the primary reason for the detection of our source, as explained above. Instead it is the unique combination of deep, wide imaging with superior image quality (PSF) including better pixel resolution of HSC data that has allowed us to discover a rare and highly compact LBG.

\subsection{Systematic uncertainties on the source size due to lens modelling}

We estimate the systematic uncertainties of lens modelling using two mock systems. The generated mocks are similar 

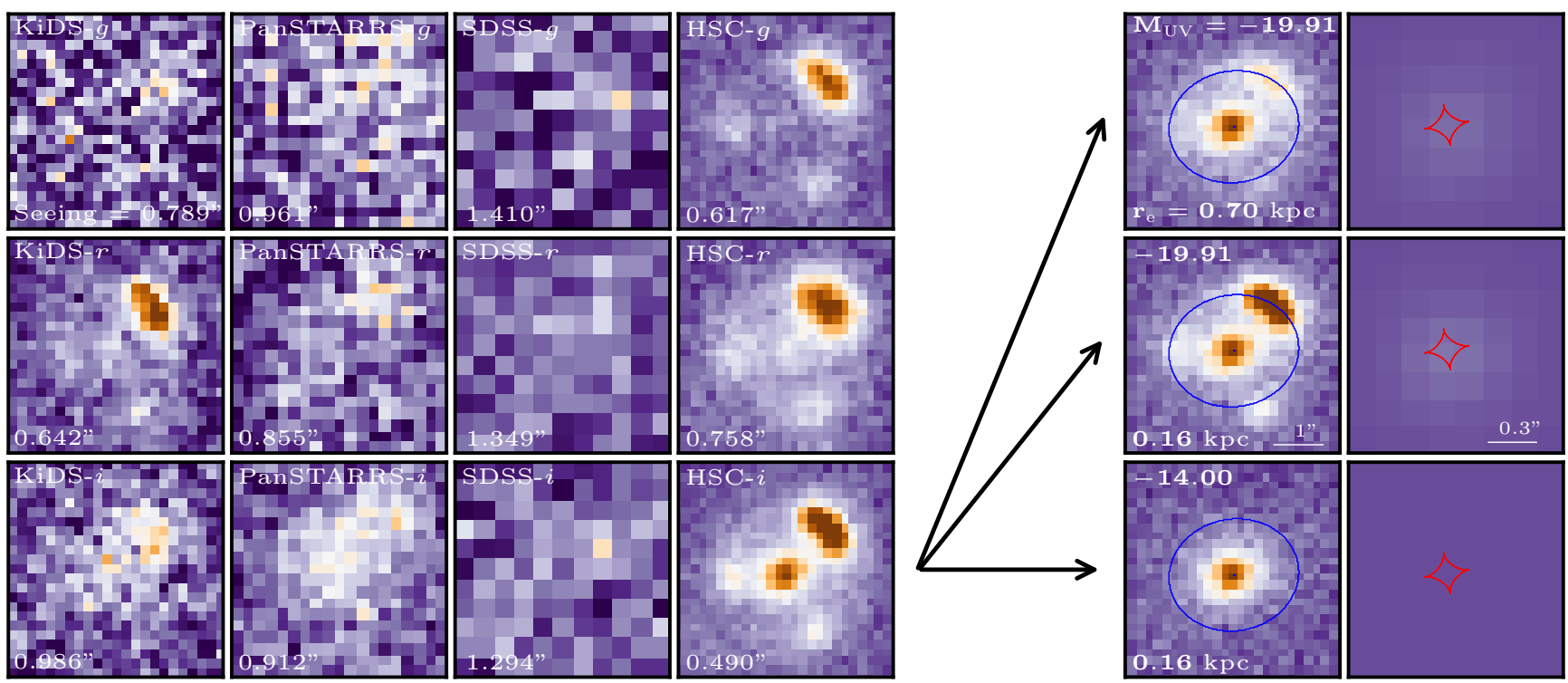

Figure 5. Left panels: HSC J0904-0102 in the footprint of other overlapping surveys (KiDS, PanSTARRS, and SDSS) with HSC Survey for $g, r$, and $i$-bands. Right panels: Lens model where lens parameters are fixed from best fitting parameters, while source was modelled by Sérsic profile with three conditions: assumed a $r_{e}$ of $\sim 0.7 \mathrm{kpc}$ (top right panel), best fitting parameters (middle right panel), and assumed a $M_{\mathrm{UV}}$ of $\sim-14$ (bottom right panel) from a $r_{e}-M_{\mathrm{UV}}$ relation as derived by S15 with the other parameters from the best-fit. Blue lines indicate critical curves. True source images for corresponding conditions are showed in right-most panels with caustic lines (red).

Table 3. Mock and best-fit model parameters.

\begin{tabular}{lrrrrr}
\hline \hline Lens Parameter & Mock1 & Model1 & Mock2 & Model2 (a) & Model2 (b) \\
\hline Velocity dispersion, $\sigma_{\text {SIE }}\left(\mathrm{km} \mathrm{s}^{-1}\right)$ & 320 & $320 \pm 1$ & - & $324 \pm 2$ & - \\
Einstein Radius, $\theta_{\text {Ein }}(\operatorname{arcsec})$ & - & - & 1.60 & - & $1.58 \pm 0.04$ \\
Ellipticity, $e$ & 0.25 & $0.24 \pm 0.01$ & 0.25 & $0.33 \pm 0.02$ & $0.25 \pm 0.03$ \\
Position Angle (degree) & -81 & -81 & 20 & $19.53 \pm 0.55$ & $19 \pm 1$ \\
Slope & 2 & 2 & 2.3 & 2 & $2.18 \pm 0.17$ \\
\hline Source Parameter & & & & & \\
\hline Magnitude & 25.15 & $25.19 \pm 0.12$ & 24.02 & $24.34 \pm 0.06$ & $23.90 \pm 0.11$ \\
Ellipticity, $e$ & 0.40 & $0.23 \pm 0.16$ & 0.4 & $0.55 \pm 0.08$ & $0.15 \pm 0.01$ \\
Position Angle $($ degree $)$ & -51 & -51 & -51 & $-56 \pm 4$ & $-77 \pm 5$ \\
Effective radius, $r_{\mathrm{e}}(\operatorname{arcsec})$ & 0.08 & $0.08 \pm 0.02$ & 0.2 & $0.25 \pm 0.03$ & $0.23 \pm 0.01$ \\
Sérsic index, $n_{\text {ser }}$ & 3.50 & $3.23 \pm 1.59$ & 1.5 & $2.82 \pm 0.54$ & $0.38 \pm 0.08$ \\
\hline
\end{tabular}

to HSC J0904-0102, that is, a single lens and a single source with redshifts and positions matched to the real lens. First, AM generated a mock lens with an SIE density profile as the lens model. The lens mass, lens ellipticity and the following source parameters were unknown to the modeller (ATJ) - flux, ellipticity, effective radius and Sérsic index. Rest of the parameters along with the choice of density profile were known to ATJ. The results of the modelling are given in Table 3. The best-fit effective radius and flux are consistent with the input values.

Next, AM generated a mock lens with a power-law density profile and a slope of 2.3 instead of the SIE (slope=2). ATJ modelled the system without any information about the choice of density profile or any of the parameter values. The corresponding best fit is given in Table 3 assuming an SIE model first and as a result the lens parameters are not fit as well. However, the source size is still consistent with the input value with flux showing some dependency on the choice of the mass density profile. In the next iteration, ATJ had the information that the density profile is not SIE. This improved the fit to the lens model parameters and the source size remained consistent with the input value within the uncertainties, as before. We point out that the source size and fluxes are constrained robustly in the above circumstances.

The source sizes in our mocks bracket the value of $\sim 0.1^{\prime \prime}$ which is the size our source would have if it were to follow Shibuya et al. relation. Since we are able to constrain the "tested" sizes with reasonable accuracy, we are confident that our inferred reff is most likely not as large as $0.1 "$. 


\section{CONCLUSION}

We report the discovery and spectroscopic confirmation of a quadruply lensed LBG at $z=3.403$. The background source is strongly lensed by a galaxy at a high redshift of $z=0.957$ with a total lensing magnification factor of 6.5. A simple SIE lens model fits the lens system well and suggests an Einstein radius, $\theta_{\text {Ein }}=1.23^{\prime \prime} \pm 0.04^{\prime \prime}\left(R_{\text {Ein }}=9.48 \pm 0.27 \mathrm{kpc}\right)$. This implies that the mass $\left(<R_{\text {Ein }}\right)$ is $5.55 \times 10^{11} M_{\odot}$. We also infer intrinsic source properties, primarily, the source size, given the magnification factor, turns out to be $0.14 \mathrm{kpc}$. In comparison with other LBGs (both lensed and unlensed) from the literature, the LBG of HSC J0904-0102 turns out to be unusually compact $(0.16 \mathrm{kpc})$ and $1 \sigma$ smaller than other LBGs (both lensed and unlensed) from the literatures. Not only the LBG is apparently faint but also is intrinsically less luminous (about $0.4 L_{*}$ ), corresponds to a star formation rate of $\sim 5 M_{\odot} \mathrm{yr}^{-1}$. Along with J1152 (More et al. 2017), another unusually compact lensed source from HSC survey, the discovery of HSC J0904-0102 hints towards the possibility that we are beginning to unearth a source population that could not be studied before.

\section{ACKNOWLEDGEMENTS}

We would like to thank Masami Ouchi and Yoshiaki Ono for useful discussions. ATJ and KTI are supported by JSPS KAKENHI Grant Number JP17H02868. MO acknowledges support from JSPS KAKENHI Grant Numbers JP15H05892 and JP18K03693. SHS thanks the Max Planck Society for support through the Max Planck Research Group. IK was supported in part by JSPS KAKENHI Grant Number JP15H05896. This work was supported in part by World Premier International Research Center Initiative (WPI Initiative), MEXT, Japan. The Hyper Suprime-Cam (HSC) collaboration includes the astronomical communities of Japan and Taiwan, and Princeton University. The HSC instrumentation and software were developed by the National Astronomical Observatory of Japan (NAOJ), the Kavli Institute for the Physics and Mathematics of the Universe (Kavli IPMU), the University of Tokyo, the High Energy Accelerator Research Organization (KEK), the Academia Sinica Institute for Astronomy and Astrophysics in Taiwan (ASIAA), and Princeton University. Funding was contributed by the FIRST program from Japanese Cabinet Office, the Ministry of Education, Culture, Sports, Science and Technology (MEXT), the Japan Society for the Promotion of Science (JSPS), Japan Science and Technology Agency (JST), the Toray Science Foundation, NAOJ, Kavli IPMU, KEK, ASIAA, and Princeton University. This paper makes use of software developed for the Large Synoptic Survey Telescope. We thank the LSST Project for making their code available as free software at http://dm.lsst.org. This paper is based [in part] on data collected at the Subaru Telescope and retrieved from the HSC data archive system, which is operated by Subaru Telescope and Astronomy Data Center at National Astronomical Observatory of Japan. Data analysis was in part carried out with the cooperation of Center for Computational Astrophysics, National Astronomical Observatory of Japan. The Pan-STARRS1 Surveys (PS1) and the PS1 public science archive have been made possible through contributions by the Institute for Astronomy, the University of Hawaii, the Pan-STARRS Project Office, the Max-Planck Society and its participating institutes, the Max Planck Institute for Astronomy, Heidelberg and the Max Planck Institute for Extraterrestrial Physics, Garching, The Johns Hopkins University, Durham University, the University of Edinburgh, the Queen's University Belfast, the Harvard-Smithsonian Center for Astrophysics, the Las Cumbres Observatory Global Telescope Network Incorporated, the National Central University of Taiwan, the Space Telescope Science Institute, the National Aeronautics and Space Administration under Grant No. NNX08AR22G issued through the Planetary Science Division of the NASA Science Mission Directorate, the National Science Foundation Grant No. AST-1238877, the University of Maryland, Eotvos Lorand University (ELTE), the Los Alamos National Laboratory, and the Gordon and Betty Moore Foundation. Based on observations obtained at the Gemini Observatory, which is operated by the Association of Universities for Research in Astronomy, Inc., under a cooperative agreement with the NSF on behalf of the Gemini partnership: the National Science Foundation (United States), National Research Council (Canada), CONICYT (Chile), Ministerio de Ciencia, Tecnología e Innovación Productiva (Argentina), Ministério da Ciência, Tecnologia e Inovação (Brazil), and Korea Astronomy and Space Science Institute (Republic of Korea). The authors wish to recognize and acknowledge the very significant cultural role and reverence that the summit of Mauna Kea has always had within the indigenous Hawaiian community. We are most fortunate to have the opportunity to conduct observations from this mountain. Based on observations made with ESO Telescopes at the La Silla Paranal Observatory under programme IDs 177.A-3016, 177.A-3017, 177.A-3018 and 179.A2004 , and on data products produced by the KiDS consortium. The KiDS production team acknowledges support from: Deutsche Forschungsgemeinschaft, ERC, NOVA and NWO-M grants; Target; the University of Padova, and the University Federico II (Naples).

\section{REFERENCES}

Adelberger K. L., Steidel C. C., Giavalisco M., Dickinson M., Pettini M., Kellogg M., 1998, ApJ, 505, 18

Adelberger K. L., Steidel C. C., Shapley A. E., Pettini M., 2003, ApJ, 584, 45

Aguado D. S., et al., 2019, ApJS, 240, 23

Aihara H., et al., 2018a, PASJ, 70, S4

Aihara H., et al., 2018b, PASJ, 70, S8

Aihara H., et al., 2019, arXiv e-prints, p. arXiv:1905.12221

Allam S. S., Tucker D. L., Lin H., Diehl H. T., Annis J., BuckleyGeer E. J., Frieman J. A., 2007, ApJ, 662, L51

Arnouts S., Cristiani S., Moscardini L., Matarrese S., Lucchin F., Fontana A., Giallongo E., 1999, MNRAS, 310, 540

Axelrod T., Kantor J., Lupton R. H., Pierfederici F., 2010, in Software and Cyberinfrastructure for Astronomy. p. 774015, doi:10.1117/12.857297

Baker A. J., Tacconi L. J., Genzel R., Lehnert M. D., Lutz D., 2004, ApJ, 604, 125

Belokurov V., et al., 2007, ApJ, 671, L9

Bolton A. S., Moustakas L. A., Stern D., Burles S., Dey A., Spinrad H., 2006, ApJ, 646, L45

Bruzual G., Charlot S., 2003, MNRAS, 344, 1000 
Cabanac R. A., Valls-Gabaud D., Jaunsen A. O., Lidman C., Jerjen H., 2005, A\&A, 436, L21

Calzetti D., Kinney A. L., Storchi-Bergmann T., 1994, ApJ, 429, 582

Cappellari M., 2017, MNRAS, 466, 798

Chambers K. C., et al., 2016, arXiv e-prints, p. arXiv:1612.05560

Coppin K. E. K., et al., 2007, ApJ, 665, 936

Dobos L., Csabai I., Yip C.-W., Budavári T., Wild V., Szalay A. S., 2012, MNRAS, 420, 1217

Ebbels T. M. D., Le Borgne J.-F., Pello R., Ellis R. S., Kneib J.-P., Smail I., Sanahuja B., 1996, MNRAS, 281, L75

Foreman-Mackey D., Hogg D. W., Lang D., Goodman J., 2013, PASP, 125, 306

Frye B. L., et al., 2007, ApJ, 665, 921

Furusawa H., et al., 2018, PASJ, 70, S3

Gemini Observatory AURA 2016, Gemini IRAF: Data reduction software for the Gemini telescopes (ascl:1608.006)

Hezaveh Y. D., Marrone D. P., Holder G. P., 2012, ApJ, 761, 20

Ilbert O., et al., 2006, A\&A, 457, 841

Ivezić Z., et al., 2008, Serbian Astronomical Journal, 176, 1

Ivezić Ž., et al., 2019, ApJ, 873, 111

Jurić M., et al., 2017, in Lorente N. P. F., Shortridge K., Wayth R., eds, Astronomical Society of the Pacific Conference Series Vol. 512, Astronomical Data Analysis Software and Systems XXV. p. 279 (arXiv: 1512.07914)

Kawanomoto S., et al., 2018, PASJ, 70, 66

Kennicutt Jr. R. C., 1998, ARA\&A, 36, 189

Koester B. P., Gladders M. D., Hennawi J. F., Sharon K., Wuyts E., Rigby J. R., Bayliss M. B., Dahle H., 2010, ApJ, 723, L73 Komiyama Y., et al., 2018, PASJ, 70, S2

Kostrzewa-Rutkowska Z., Wyrzykowski Ł., Auger M. W., Collett T. E., Belokurov V., 2014, MNRAS, 441, 3238

Kuijken K., et al., 2019, A\&A, 625, A2

Marques-Chaves R., et al., 2017, ApJ, 834, L18

Mason R. E., Côté S., Kissler-Patig M., Levenson N. A., Adamson A., Emmanuel C., Crabtree D., 2014, in Observatory Operations: Strategies, Processes, and Systems V. p. 914910 (arXiv: 1408.5916), doi:10.1117/12.2057145

Mehlert D., et al., 2001, A\&A, 379, 96

Miyazaki S., et al., 2018, PASJ, 70, S1

More A., et al., 2017, MNRAS, 465, 2411

Oguri M., 2010, PASJ, 62, 1017

Oguri M., et al., 2018, PASJ, 70, S20

Oldham L., et al., 2017, MNRAS, 465, 3185

Ono Y., et al., 2018, PASJ, 70, S10

Peng C. Y., Ho L. C., Impey C. D., Rix H.-W., 2002, AJ, 124, 266

Pettini M., Steidel C. C., Adelberger K. L., Dickinson M., Giavalisco M., 2000, ApJ, 528, 96

Pettini M., Rix S. A., Steidel C. C., Adelberger K. L., Hunt M. P., Shapley A. E., 2002, ApJ, 569, 742

Quider A. M., Pettini M., Shapley A. E., Steidel C. C., 2009, MNRAS, 398, 1263

Quider A. M., Shapley A. E., Pettini M., Steidel C. C., Stark D. P., 2010, MNRAS, 402, 1467

Reddy N. A., Steidel C. C., 2009, ApJ, 692, 778

Richard J., Stark D. P., Ellis R. S., George M. R., Egami E., Kneib J.-P., Smith G. P., 2008, ApJ, 685, 705

Richard J., Jones T., Ellis R., Stark D. P., Livermore R., Swinbank M., 2011, MNRAS, 413, 643

Sánchez-Blázquez P., et al., 2006, MNRAS, 371, 703

Sawicki M., Thompson D., 2006, ApJ, 642, 653

Shapley A. E., Steidel C. C., Pettini M., Adelberger K. L., 2003, ApJ, 588, 65

Shibuya T., Ouchi M., Harikane Y., 2015, ApJS, 219, 15

Siana B., et al., 2009, ApJ, 698, 1273

Smail I., et al., 2007, ApJ, 654, L33
Sonnenfeld A., Gavazzi R., Suyu S. H., Treu T., Marshall P. J., 2013a, ApJ, 777, 97

Sonnenfeld A., Treu T., Gavazzi R., Suyu S. H., Marshall P. J., Auger M. W., Nipoti C., 2013b, ApJ, 777, 98

Sonnenfeld A., et al., 2018, PASJ, 70, S29

Sonnenfeld A., Jaelani A. T., Chan J. H. H., More A., Suyu S. H., Wong K. C., Oguri M., Lee C.-H., 2019, arXiv e-prints, p. arXiv:1904.10465

Stark D. P., Ellis R. S., Richard J., Kneib J.-P., Smith G. P., Santos M. R., 2007, ApJ, 663, 10

Steidel C. C., Adelberger K. L., Dickinson M., Giavalisco M., Pettini M., Kellogg M., 1998, ApJ, 492, 428

Steidel C. C., Shapley A. E., Pettini M., Adelberger K. L., Erb D. K., Reddy N. A., Hunt M. P., 2004, ApJ, 604, 534

Tody D., 1986, in Crawford D. L., ed., Proc. SPIEVol. 627, Instrumentation in astronomy VI. p. 733, doi:10.1117/12.968154

Tody D., 1993, in Hanisch R. J., Brissenden R. J. V., Barnes J., eds, Astronomical Society of the Pacific Conference Series Vol. 52, Astronomical Data Analysis Software and Systems II. p. 173

Wolf J., Martinez G. D., Bullock J. S., Kaplinghat M., Geha M., Muñoz R. R., Simon J. D., Avedo F. F., 2010, MNRAS, 406, 1220

Wong K. C., et al., 2018, ApJ, 867, 107

Yee H. K. C., Ellingson E., Carlberg R. G., 1996, ApJS, 102, 269

This paper has been typeset from a $\mathrm{T}_{\mathrm{EX}} / \mathrm{LAT} \mathrm{EX}$ file prepared by the author. 